\title{
Interest of Screening and Management for Sleep Disorders in the Drug-Resistant Depressive Syndrome
}

\section{Sandrine Pradel-Neuman and Eric Neuman*}

Centre Vitruve, 23 rue Galilée, Paris, France

*Corresponding Author: Eric Neuman, Centre Vitruve, 23 rue Galilée, Paris, France.
Received: February 17, 2020

Published: May 11, 2020

(c) All rights are reserved by Sandrine

Pradel-Neuman and Eric Neuman.

\begin{abstract}
During the last two years, in a multidisciplinary psychiatric practice, a psychiatrist assisted with a sleep therapist decided to demonstrates that the management of secondary insomnia (induced by sleep apnea/hypopnea syndrome (SAHS)) and some other primary sleep disorders, with specific sleep therapy can reduce or sometimes even stop antidepressant treatments when a welldefined protocol is followed.

Their results confirmed that in some of their patients there may be SAHS in drug-resistant depressions. This point out the fact any depression syndromes are due to or aggravated by insomnia induced by SAHS. Taking care of it may replace antidepressants.

Keywords: Sleep Disorders; Depressive Syndrome; Drug Resistance
\end{abstract}

\section{Introduction}

In a multidisciplinary psychiatric practice, many outpatients consult for sleep disorders of primary or secondary origin. In recent years, we have observed an increasing number of cases of sleep apnea/hypopnea syndrome (SAHS) and other primary sleep disorders, convincing us to proceed with a more systematic search for these sleep disorders in our anxio-depressive patients, in particular, those resistant to pharmacological treatment.

Existing literature [1-3] which somewhat argue for prevalence, cause and treatment of depression in obstructive sleep apnea is copious but fragmented. It seemed to us to be interesting to describe some of our clinical experience for underlying relationship between sleep disorders shown by our patients and the impact of treatments in patients with chronic and drug resistant depression.

In French literature, interest of link between sleep disorders and depression is somewhat recent [4-8], but also target the possible role of obstructive chronic apnea syndrome in developing and aggravating mood disorders.

So, we naturally hypothesized that in some of our patients there may be SAHS in drug-resistant depressions. We tested this hypothesis by setting up a paramedical sleep consultation, using a protocol of therapeutic educational method of medical following, a procedure approved by the French Regional Health Agency (Agence régionale de santé - ARS), integrating a sleep therapist under the responsibility of a psychiatric coordinator.

Each patient seen at least once in a psychiatric consultation and presenting with proven sleep or vigilance complaints was addressed to the sleep therapist.

\section{Method}

We include in this cases study all patients who consult the psychiatrist practitioner of our medical office during last two years for depression chronic episode, with drug resistance, that means who have not enough good clinical or social response after at least two antidepressant treatments at high dosage prescribed for at least 8 weeks.

Then, we describe below details for three patients, who are particularly representative according our experience.

The procedure had three steps:

1. The first consisted of an overall assessment of the patient's personal, social and professional characteristics.

2. The second consisted of filling out specialized questionnaires (Epworth Scale) and a sleep diary. Depending on the results, a ventilatory polygraph (VP) screening test was performed, recording only respiratory events $[9,10]$.

3. Finally, improvements in the patient following proposed strategies were evaluated by the ISI scale (Insomnia Severity Index) and the Pichot Fatigue Scale.

For a period of 1 year, 127 patients were included in the study, aged 7 to 70,49 male and 78 female, with chronic insomnia and/or circadian rhythm disorders. Seventy-nine of them were diagnosed with drug-resistant depression.

\section{Results and Discussion}

Of the 79 subjects getting a VP, 53 had SAHS. Of these 53 patients, 32 had severe SAHS with an apnea/hypopnea index (AHI) 
$>30 / \mathrm{h}$ that required continuous positive airway pressure (CPAP) therapy. Of these 53 patients, 26 had an additional polysomnography (PSG) examination. Eleven of them were treated by an ENT (surgical intervention) for upper airway permeability issues, and the other patients were treated for periodic limb movement (PLM).

Distribution of 79 patients with chronic drug-resistant depression who had an VP.

One month later, a visit to the sleep therapist was organized to assess insomnia. Subjects reported a marked improvement in their sleep.
Three months later, out of the 79 depressed patients with chronic insomnia who benefited from sleep disorder management, 22 no longer suffered from insomnia and the others complained less about it, with a corresponding notable decrease in their antidepressant treatment.

Overview of improvements following work with sleep therapist.

To follow-up this regular therapy, a consultation with the psychiatrist was organized to assess their psychological state.

\begin{tabular}{|l|c|c|c|c|c|}
\hline $\begin{array}{c}\text { Number of } \\
\text { positive VPs }\end{array}$ & $\begin{array}{c}\text { Number of } \\
\text { additional } \\
\text { PSG }\end{array}$ & $\begin{array}{c}\text { Number of patients with severe } \\
\text { SAHS with an AHI > 30 and thus } \\
\text { treated with CPAP }\end{array}$ & $\begin{array}{c}\text { Number of patients with } \\
\text { PLM and restless leg } \\
\text { syndrome }\end{array}$ & $\begin{array}{c}\text { Number of } \\
\text { patients treated } \\
\text { by ENT }\end{array}$ & $\begin{array}{c}\text { Number of patients } \\
\text { treated for PLM and } \\
\text { SAHS }\end{array}$ \\
\hline 53 & 26 & 32 & 25 & 11 & 11 \\
\hline
\end{tabular}

Table 1

\begin{tabular}{|c|c|c|c|c|c|c|}
\hline Patients with severe SAHS (53) & $\begin{array}{c}\text { Epworth } \\
\text { Scale } \\
\text { average }\end{array}$ & $\begin{array}{c}\text { Pichot } \\
\text { Average }\end{array}$ & $\begin{array}{c}\text { ISI } \\
\text { average }\end{array}$ & $\begin{array}{c}\text { Number of } \\
\text { patients with } \\
\text { improvements }\end{array}$ & $\begin{array}{c}\text { Number of } \\
\text { patients with less } \\
\text { insomnia }\end{array}$ & $\begin{array}{c}\text { Number of } \\
\text { patients using } \\
\text { hypnotics }\end{array}$ \\
\hline Before treatment & 11 & 21 & 18 & 79 & 0 & 52 \\
\hline One month after treatment & 9 & 15 & 14 & 32 & 22 & 16 \\
\hline After 3 months of treatment & 4 & 8 & 7 & 54 & 65 & 22 \\
\hline
\end{tabular}

Table 2

To develop our suggestion, several examples are presented below.

Case study 1: Mr. Th.

Psychiatric description

A 52-year-old patient had been suffering from a depressive state for several years with psychomotor retardation, abulia, lack of enthusiasm, loss of motivation, and chronic sadness.

Judged "not funny" by his family and colleagues, he noted a decline in performance, was disappointed in himself, was constantly fatigued and unable to recover even during vacations. There were few, if any, effects from several prescribed antidepressants (Fluoxetine, Venlafaxine, Mirtazapine-worse, Escitalopram).

\section{Assessment by the sleep therapist}

- $\quad$ Sleep assessment and description of the patient's agenda: His sleep was fragmented, phase delayed, with daytime sleepiness at 17 on the Epworth scale (normal value $<8$ ) and he had poor sleep hygiene.

- Morning symptoms: Headaches, not feeling refreshed upon waking.

Mr. Th. S. sleep diary before care

(Figure 1)

Prescription: ventilatory polygraph

The VP analysis showed an apnea/hypopnea index at 40 per hour (normal value $<5 / \mathrm{h}$ ).

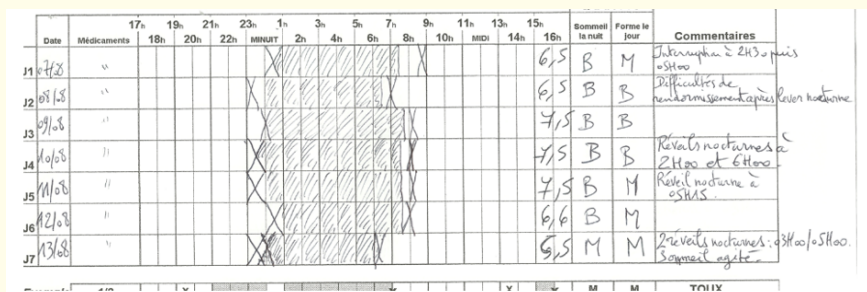

Figure 1

Based on these results, a comprehensive treatment plan was proposed to the subject.

At the same time that CPAP treatment began, cognitive educational accompaniment twice a month was used to improve sleep hygiene.

\section{Results}

After 6 months of treatment of CPAP (the patient has been already seen after 1 month and then after 3 months) by both the psychiatrist and the sleep therapist:

- $\quad$ The patient had good CPAP compliance, sleep hygiene was respected, the number of hours of sleep had increased, his sleep was again pleasant, he was no longer tired in the morning, no longer had headaches and felt more dynamic.

- $\quad$ The Epworth Scale had returned to normal at 8. He felt more awake during the day. 
- The psychiatrist consequently decreased antidepressant dosage (Escitalopram initially prescribed to the dose of $15 \mathrm{mg}$ be reduced to $5 \mathrm{mg}$ per day) at the end of these 6 months and stopped it entirely after one year.

Mr. Th. S. sleep schedule after treatment

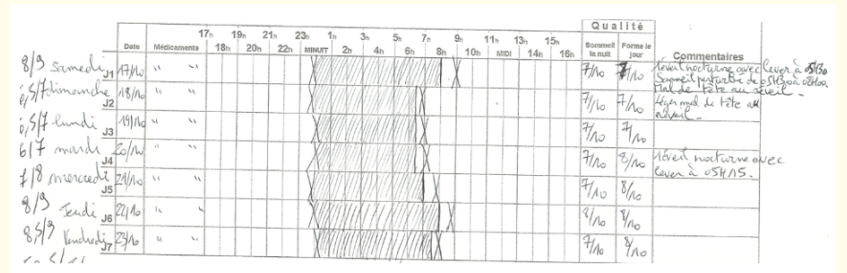

Figure 2

Case study 2: Ms. V. L.

Psychiatric description

A 38-year-old patient had isolated herself at home for more than 15 years with psychomotor retardation, «melancholiform» sadness, abulia, no initiative, severe procrastination, social isolation, lack of leisure... She was in a household with a «geek» who was thus very tolerant! She felt constantly fatigued and felt guilty.

Antidepressants had almost no effect (almost all had been tested), nor had regulators and even less the neuroleptics that had been prescribed for years.

\section{Assessment by the sleep therapist}

- Sleep assessment and description of subject's sleep diary: Her sleep was fragmented. She had daytime drowsiness with an Epworth scale score of 13. She lived with the shutters closed day and night. The patient followed no schedule for going to bed or getting up. She had very poor sleep hygiene.

- Morning symptoms: sleep deemed non-restorative, headaches.

Ms. V.L.'s initial sleep diary

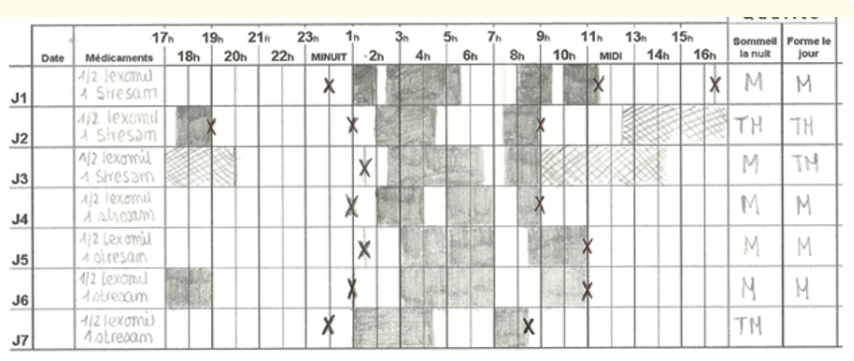

Figure 3

\section{Prescription: VP}

The VP analysis showed a light SAHS with many inspiratory flow limitations. It was sent to an ENT who diagnoses a significant deviation of the nasal septum. She then benefited from specialized surgery and CBT to reorganize her biological clock.

\section{Results}

After 7 therapy sessions of educational accompaniment therapy spread over 3 months, both by the psychiatrist and by the sleep therapist:

- $\quad$ Sleep was adapted again. She had much better sleep hygiene (going to bed and getting up at regular times) respecting the circadian cycle, including opening and closing the shutters.

- $\quad$ There was a clear decrease in fatigability. She regained dynamism, her headaches were gone, and she felt cooler in the morning when waking up.

- The psychiatrist was able to stop the psychoactive treatment (which associated paroxetine (as an antidepressant, at the dose of $20 \mathrm{mg}$ ) and aripiprazole (prescribed as a mood regulator, at the initial dose of $5 \mathrm{mg}$ ).

Case study 3: Ms. S.P

Psychiatric description

A habitually very active 48-year-old woman experienced a major depressive episode of severe intensity ( $2^{\text {nd }}$ relapse in 3 years) with psychomotor retardation, hypersomnia refuge, abulia, loss of motivation and pathological sadness.

Exhibiting unusual social withdrawal, she no longer assumed her family responsibilities and her work stoppage made her feel guilty.

She was experiencing daytime drowsiness and nocturnal insomnia, constant fatigability, without recovery despite treatment with several antidepressants (including Anafranil and a euthymic prescribed at the time of this episode due to suspicion of a bipolar disorder).

\section{Mrs. S.P.'s initial sleep diary}

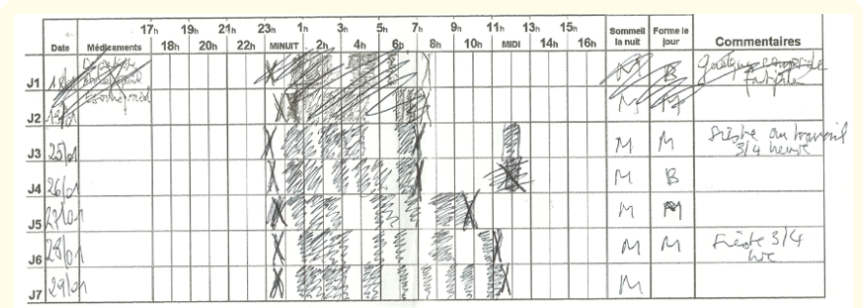

Figure 4

Assessment by the sleep therapist

- $\quad$ Sleep assessment and sleep schedule description: Fragmented sleep, excessive daytime sleepiness with an Epworth Scale at 17.

- Morning symptoms: Fatigue, memory and concentration problems, sensitive to lack of light, sad.

- $\quad$ Prescription of a PSG: It showed PLM and restless leg movements. 
- $\quad$ Prescription of martial blood test: Revealed that she was deficient in iron. Based on these elements, the psychiatrist prescribed treatment with Pramipexol and iron.

Results

After 3 sessions of monitoring spread over 3 months:

- Her sleep was adjusted with diminished fatigability. She regained dynamism, no longer felt daytime sleepiness with an Epworth Scale at 8/24.

- The treatment was adapted leading to a decrease of the antidepressant.

Case study 4: Mr. V.E.

Psychiatric description

The 61-year-old patient was sad, addict to sleeping pills (Zolpidem since the age of 33), weary, had psychomotor retardation, was constantly fatigable, and could not recover even during holidays.

\section{Therapeutic sleep assessment}

- Assessment of sleep and sleep schedule: He complained of poor-quality sleep, with scary night awakenings. The patient was taking sleeping pills to fall asleep faster but remained tired in the morning. He also complained of memory and concentration problems and excessive daytime sleepiness with an Epworth Scale of 17.

- Morning symptoms: headache, dry mouth, irritability.

- $\quad$ Sleep Diary: It shown sleep latency and insomnia.

Mr V.E.'s initial diary

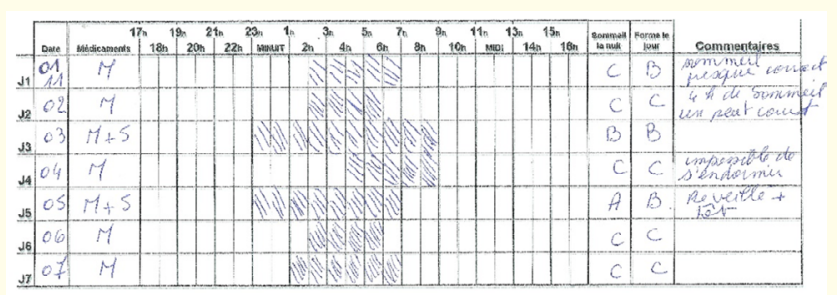

Figure 5

Prescription of a ventilatory polygraph

It showed an AHI result at $41 / \mathrm{h}$, indicating the prescription of CPAP treatment.

Results

- $\quad$ After 1 month under CPAP, with good compliance, the patient did not feel tired in the morning and headaches were gone. He was ready to reduce his sleeping pill usage.

- $\quad$ After 3 months of follow-up (9 sessions), the patient was no longer taking sleeping pills, felt well, did not suffer from daytime drowsiness and had no trouble falling asleep. Color returned to his facial complexion.

Mr V.E.'s sleep diary after treatment

(Figure 6)

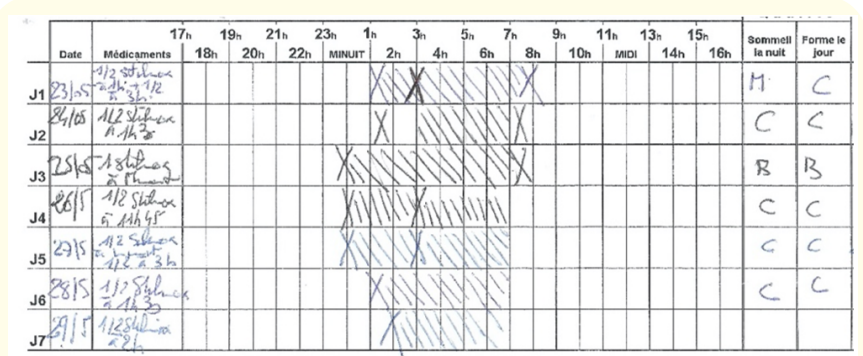

Figure 6

\section{Conclusion}

This study demonstrates that the management of insomnia with specific sleep therapy can replace doses or sometimes even stop antidepressant treatments when a well-defined protocol is followed. We therefore recommend performing a routine VP screening in subjects of all ages with depressive syndrome, especially when it is drug-resistant and when the patient has experienced longstanding sleep disorders [11-16]

\section{Bibliography}

1. Ejaz SM., et al. "Obstructive Sleep Apnea and Depression. A Review”. Innovations in Clinical Neuroscience 8.8 (2011): 17-25.

2. Harris M., et al. "Obstructive sleep apnea and depression". Sleep Medicine Reviews 13.6 (2009): 437-444.

3. Schröder CM and O'Hara R. “Depression and Obstructive Sleep Apnea (OSA)”. Annals of General Psychiatry 4 (2005): 13.

4. Beck L., et al. "Sleep disorders: Sociodemographics and psychiatric comorbidities in a sample of 14,734 adults in France (Baromètre santé INPES)". Revue Neurologique 165.11 (2009): 933-942.

5. Gharsalli H., et al. "Caractéristique des patients SAOS dépressifs". Médecine du Sommeil 16.1 (2019): 45.

6. Mollo JL. "Insomnie et syndrome d'apnée obstructive du sommeil: liaison dangereuse". Médecine du Sommeil 11.4 (2014): 197-205.

7. Royant-Parola S. "Depression et SAS: une intrication sans fin; Depression and sleep apnéea syndrome an endless entanglement”. Médecine du Sommeil 10.1 (2013): 29-31.

8. Royant -Parola S. "Depression et troubles cognitifs dans le SAS; Depression and cognitive disorders en SAS”. Revue des Maladies Respiratoires 23.2 (2006): 94-96.

9. Chalumeau F. "SAOS de l'Adulte: Les Explorations du Sommeil par PGV et PSG". La lettre d'Oto-rhino-larymgologie et de Chirurgie (2012).

10. Launois S and Rey M. "Place des différents enregistrements au cours du sommeil: résumé du rapport de l'évaluation de l'HAS. Practice guidelines for the various sleep recordings, summary of HAS evaluation report". Médecine du Sommeil 10.1 (2013): 6-11. 
11. Bahammam AS., et al. "Comorbid depression in obstructive sleep apnea: an under-recognized association". Sleep Breath 20.2 (2016): 447-456.

12. Fresco JP., et al. "Somnolence diurne excessive et dépression: données polysomnographiques. Excessive daytime sleepimen and depression polysomnographical data". Médecine du Sommeil 10.1 (2013): 19-28.

13. Leclair-Visonneau L., et al. “Comment poser le diagnostic d'un syndrome des jambes sans repos? How to diagnose restless legs syndrome?" Médecine du Sommeil 16.2 (2019): 97-105.

14. Limousin N., et al. "Traitement du syndrome des jambes sans repos nouvellement diagnostiqué; Treatment of newly diagnosed restless legs syndrome". Médecine du Sommeil 16.2 (2019): 106-113.

15. Micoulaudet JA., et al. "Réalization and interpretation of sleep exploration for sleep disorders with mental disorders comorbidities". Annales Médico-psychologiques, Revue Psychiatrique 177.5 (2019): 468-482.

16. Neuman E and Neuman-Pradel S. "Mes nuits sans sommeil, tout comprendre sur l'insomnie; my nights without sleeping, all understand about insomnia". Editions Grégo-Vernazobres, Paris (2017): 190.

\section{Assets from publication with us}

- Prompt Acknowledgement after receiving the article

- Thorough Double blinded peer review

- Rapid Publication

- Issue of Publication Certificate

- High visibility of your Published work

Website: https://www.actascientific.com/

Submit Article: https://www.actascientific.com/submission.php Email us: editor@actascientific.com

Contact us: +919182824667 